\title{
Usefulness of End-to-Side Bridging Anastomosis of Sural Nerve to Tibial Nerve : An Experimental Research
}

\author{
Soner Civi, M.D., ${ }^{1}$ Emre Durdag, M.D., ${ }^{1}$ Murat Hamit Aytar, M.D., ${ }^{2}$ Ozgur Kardes, M.D., ${ }^{1}$ Figen Kaymaz, M.D., ${ }^{3}$ Sukru Aykol, M.D. ${ }^{4}$ \\ Department of Neurosurgery, Başkent University Faculty of Medicine, Adana, Turkey \\ Department of Neurosurgery, ${ }^{2}$ Acibadem University Vocational School of Health Services, Ístanbul, Turkey \\ Department of Histology and Embryology, ${ }^{3}$ Hacettepe University Faculty of Medicine, Ankara, Turkey \\ Department of Neurosurgery, ${ }^{4}$ Gazi University Faculty of Medicine, Ankara, Turkey
}

Objective : Repair of sensorial nerve defect is an important issue on peripheric nerve surgery. The aim of the present study was to determine the effects of sensory-motor nerve bridging on the denervated dermatomal area, in rats with sensory nerve defects, using a neural cell adhesion molecule (NCAM).

Methods : We compared the efficacy of end-to-side (ETS) coaptation of the tibial nerve for sural nerve defect repair, in 32 SpragueDawley rats. Rats were assigned to 1 of 4 groups : group A was the sham operated group, group B rats had sural nerves sectioned and buried in neighboring muscles, group C experienced nerve sectioning and end-to-end (ETE) anastomosis, and group D had sural nerves sectioned and ETS anastomosis was performed using atibial nerve bridge. Neurological evaluation included the skin pinch test and histological evaluation was performed by assessing NCAM expression in nerve terminals.

Results : Rats in the denervated group yielded negative results for the skin pinch tests, while animals in the surgical intervention groups (group C and D) demonstrated positive results. As predicted, there were no positively stained skin specimens in the denervated group (group B); however, the surgery groups demonstrated significant staining. NCAM expression was also significantly higher in the surgery groups. However, the mean NCAM values were not significantly different between group $C$ and group D.

Conclusion : Previous research indicates that ETE nerve repair is the gold standard for peripheral nerve defect repair. However, ETS repair is an effective alternative method in cases of sensorial nerve defect when ETE repair is not possible.

Key Words : Nerve bridging · Sensory nerve defect · End-to-side coaptation · Neural cell adhesion molecules.

- Received : October 17, 2016 •Revised : December 17, 2016 •Accepted : February 13, 2017

- Address for reprints : Soner Civi, M.D.

Department of Neurosurgery, Başkent University Faculty of Medicine, DadaloğluMah, Şirinevler 2591.sk no:4/A 01250 Yüreğir, Adana, Turkey Tel : +90-532-402-51-45, Fax : +90-322-327-12-74, E-mail : sonercivi@yahoo.com

This is an Open Access article distributed under the terms of the Creative Commons Attribution Non-Commercial License (http://creativecommons.org/licenses/by-nc/4.0) which permits unrestricted non-commercial use, distribution, and reproduction in any medium, provided the original work is properly cited. 


\section{INTRODUCTION}

End-to-side (ETS) nerve anastomosis provides reinnervation of the denervated area by collateral formation. In this technique, which has existed for at least a century, the injured nerve is coapted to the side of an uninjured nerve via its stump ${ }^{20)}$. ETS anastomosis is primarily used for repairing nerve injuries that have a gap between proximal and distal stumps, and when end-to-end (ETE) anastomosis of the injury is not possible. Developments in microsurgical techniques and popularization of both nerve grafting and primary repair methods have decreased the use of ETS anastomosis. In the early 1990's, use of the ETS technique increased, primarily due to histological and electrophysiological findings by Viterbo, Trindade, Hoshino, and Mazzoni showing reinnervation of the damaged nerve ${ }^{21,22)}$. ETS anastomosis renders long nerve grafts unnecessary and is especially easy to perform in the absence of a proximal stump or when there is a long gap between the proximal and distal ends of the injured nerve ${ }^{4,19,20)}$.

The whole integrity of the nervous system is important for the modern brain surgery. In the light of this insight, the importance of the repair of sensory nerve defects cannot be denied. In parallel with this requirement, the articles on the repair techniques of sensory nerve defects have begun to appear in the literature, especially for the past 10 years ${ }^{19,20)}$. The efficacy of ETS anastomosis has been identified when the nerve defect is large, particularly in digital nerve lacerations ${ }^{19)}$. Based on our present knowledge, however, no technique relating to the use of a nerve with motor functions as a bridge in sensory nerve defects has been identified.

We investigated the efficacy of a motor nerve bridge to repair a sensory nerve defect. We used the tibial nerve of the rat as the bridge for a sural nerve defect, by performing ETS anastomosis. Since the sural nerve is a sensory nerve, in order to assess the effects of denervation and re-innervation (after coaptation) at the end organ - which is the skin of the animalwe used a neural cell adhesion molecule (NCAM) as the marker.

\section{MATERIALS AND METHODS}

\section{Animals}

This study was performed at Gazi University School of Me- dicine Experimental Research Center, and was approved by the local ethics committee. All animals received humane care in compliance with the "Principles of Laboratory Animal Care" formulated by the National Society for Medical Research and the "Guide for the Care and Use of Laboratory Animals" prepared by the Institute of Laboratory Animal Resources and published by the National Institutes of Health (USA).

Thirty-two adult male Sprague-Dawley rats weighing 300$350 \mathrm{~g}$ were randomly divided into 4 groups $(\mathrm{n}=8)$ as follows : 1) group A : sham operated group, 2) group B : sural nerves were sectioned and proximal and distal stumps were buried in neighboring muscles, 3) group C : Sural nerves were sectioned and ETE anastomosis was performed, and 4) group D : sural nerves were sectioned and proximal and distal stumps were sutured to the intact tibial nerve using an ETS technique with $5 \mathrm{~mm}$ spacing.

\section{Surgical procedure}

Rats were housed at room temperature and had free access to food and tap water. They were anesthetized by intramuscular injection of $50 \mathrm{mg} / \mathrm{kg}$ ketamine (Ketalar; Pfizer, Kırklareli, Turkey) and $5 \mathrm{mg} / \mathrm{kg}$ xylazine (Rompun; Bayer, Kansas, KS, USA). Arterial pressure and heart rate were constantly monitored; breathing was unassisted.

After administration of anesthesia, the right hind limbs were prepared for surgery using a sterile technique. Skin incision was followed by dissection of the gluteal muscles in order to expose the sciatic nerve, including the tibial, peroneal, and sural branches, under a surgical microscope (Opmi 9-FC; Carl Zeiss AG, Oberkochen, Germany). No further intervention was performed in group $\mathrm{A}$, and the incisions were closed with sutures. In group B, the sural nerve was sharply severed distal to its bifurcation from the sciatic nerve. The free proximal and distal stumps of the sural nerve were buried into the gluteal muscles prior to suturing. In group C, ETE anastomosis of the proximal and distal stumps was performed after sectioning the sural nerve. In group D, the sural nerve was sectioned and a $5 \mathrm{~mm}$ segment was excised from the distal stump, in order to simulate a nerve defect. The proximal and distal ends were then sutured to the intact tibial nerve using an ETS technique. The epineurium of the sural and tibial nerves was removed at the site of coaptation. Anastomoses were performed using 10-0 nylon sutures (Fig. 1). 

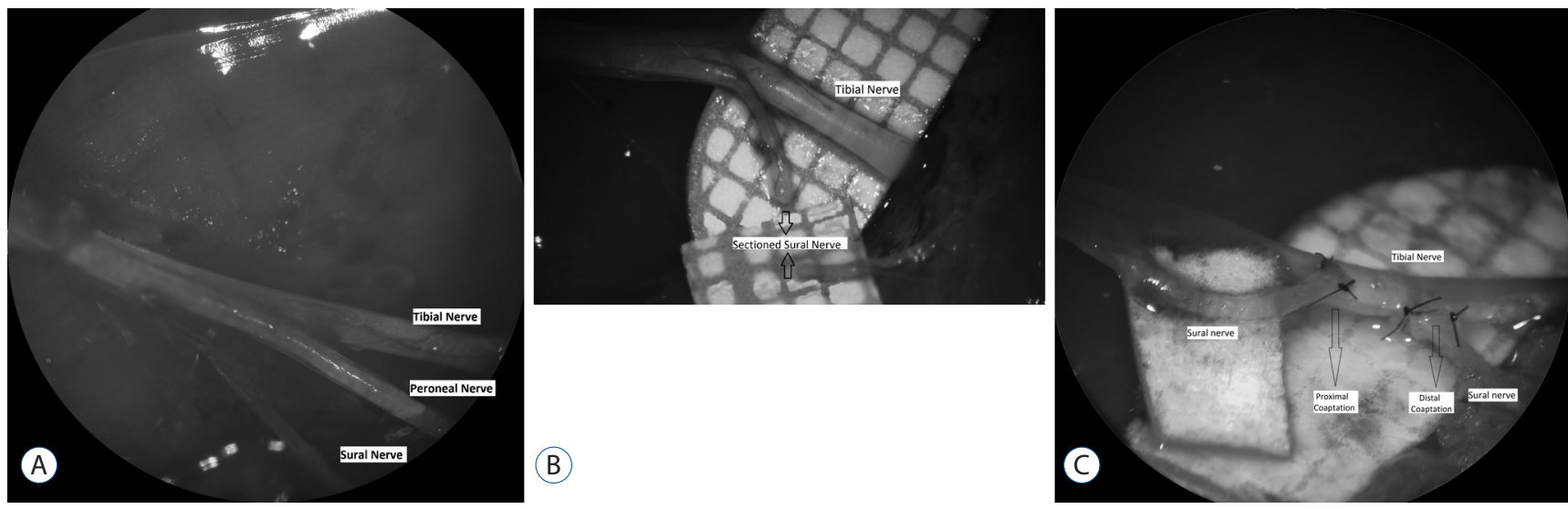

Fig. 1. A : The divisions of sciatic nevre under operation microscope. B : Peroneal division is spared below with metric paper, sural nerve is sectioned. Proximal and distal endings of sectioned sural nerve is demostrated by arrows. C : Sural stumps are coapted to tibial nerve by ETS fashion. Proximal and distal coaptation of sural nerve is demostrated by arrows. ETS : end-to-side.

\section{Sensory assessment}

In order to evaluate the functional status of the sural nerve at its dermatome in the right hind paw, the skin pinch test was performed. The skin of the right hind paw was stimulated using an aneurysm clip (Yasargil Aneurysm Clip, closing force $188 \mathrm{gr} / \mathrm{cm}^{2}$ ) and positive responses were indicated by foot withdrawal. All animals were tested on postoperative day 1 and at weeks 4,8 , and 12 .

\section{Histological evaluation}

Histological evaluations were performed by a specialist (F.K.) who was blinded to the animal's group status. Rats were sacrificed after 12 weeks, using high dose intraperitoneal pentobarbital $(200 \mathrm{mg} / \mathrm{kg})$ and the sural nerve dermatome was harvested (Fig. 2) 2 .6).

Tissue samples were fixed in formaldehyde (10\%, pH 7.4), and then embedded in paraffin. A microtome was used to cut $4 \mu \mathrm{m}$ sections, and every 6th section was mounted on a glass slide. After removing the paraffin, the sections were treated with a polyclonal antibody for NCAM (anti-neural cell adhesion molecule, Cat No AB5032; Chemicon, Billerica, MA, USA) and stained using the indirect ABC method. Five randomly selected representative fields were evaluated at $\times 20$ magnification and the number of NCAM positive free nerve endings were recorded. The mean value was calculated from measurements of the 5 fields and recorded as the number of NCAM-positive free nerve endings.
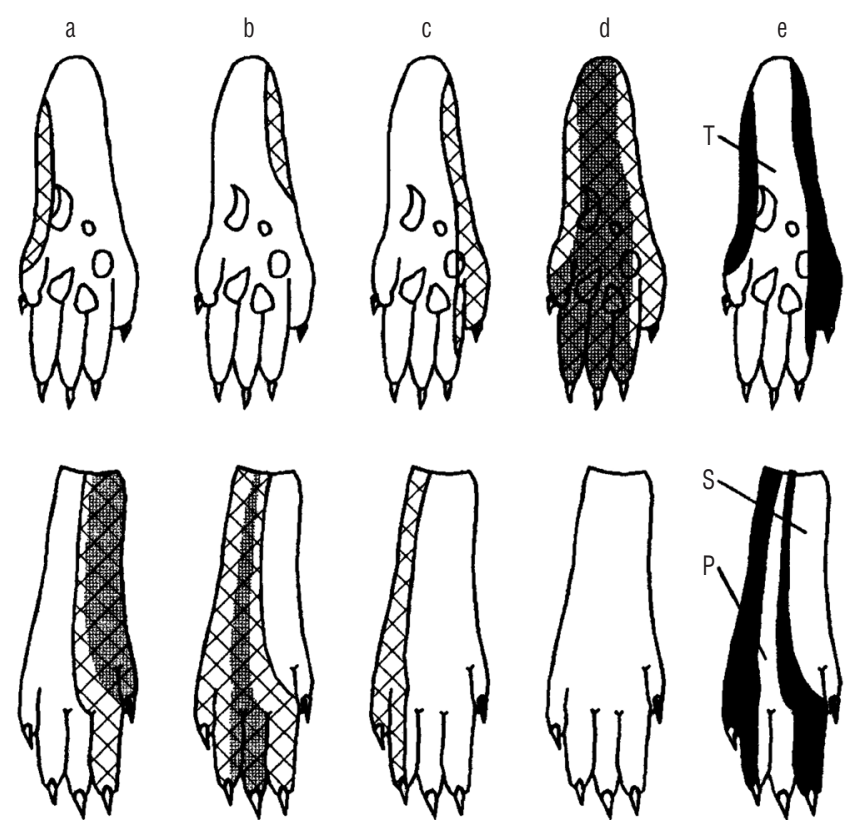

Fig. 2. A typical compound picture of nociceptive innervation fields of the examined peripheral nerves. Saphenous nerve (a), peroneal nerve (b), sural nerve (c), tibial nerve (d), and compound innervation of nociceptive dermatomes (e). $\mathrm{T}$ : tibial nerve, $\mathrm{S}$ : sural nerve, $\mathrm{P}$ : peroneal nerve.

\section{Statistical analysis}

Statistical analyses were performed using the SPSS statistical software package (version 17.0; SPSS Inc., Chicago, IL, USA). When continuous variables were normally distributed, they are presented as the mean \pm standard deviation $(p>0.05$ in Kolmogorov-Smirnov test or Shapiro-Wilk test, $\mathrm{n}<30$ ). When continuous variables were not normally distributed, they are reported as median values. Between-group comparisons of 
non-normally distributed data were conducted using the Kruskal-Wallis test. When analysis of variance (ANOVA) tests were significant, Tukey's post-hoc tests were conducted. Values of $p<0.05$ were considered statistically significant.

\section{RESULTS}

\section{Sensory assessment}

All rats in group A demonstrated positive responses to the pinch test, whereas rats in group B demonstrated negative responses at all tested time intervals.

Rats in groups $\mathrm{C}$ and $\mathrm{D}$ demonstrated increasing positive responses across time intervals. Fig. 3 indicates the number of rats with positive pinch test responses across time intervals. The difference between groups C and D was not statistically significant.

\section{Histological evaluation}

The median number of areas positively stained with anti-NCAM was 27.4 (18.4-32.2) for group A, 8.5 (5.4-11.4) for group B, 16.5 (10.4-21.6) for group C, and 13.5 (6.6-17.6) for group D. The mean number of areas positively stained with anti-NCAM was statistically different between groups. Tukey's posthoc analyses revealed that the values for all surgical groups

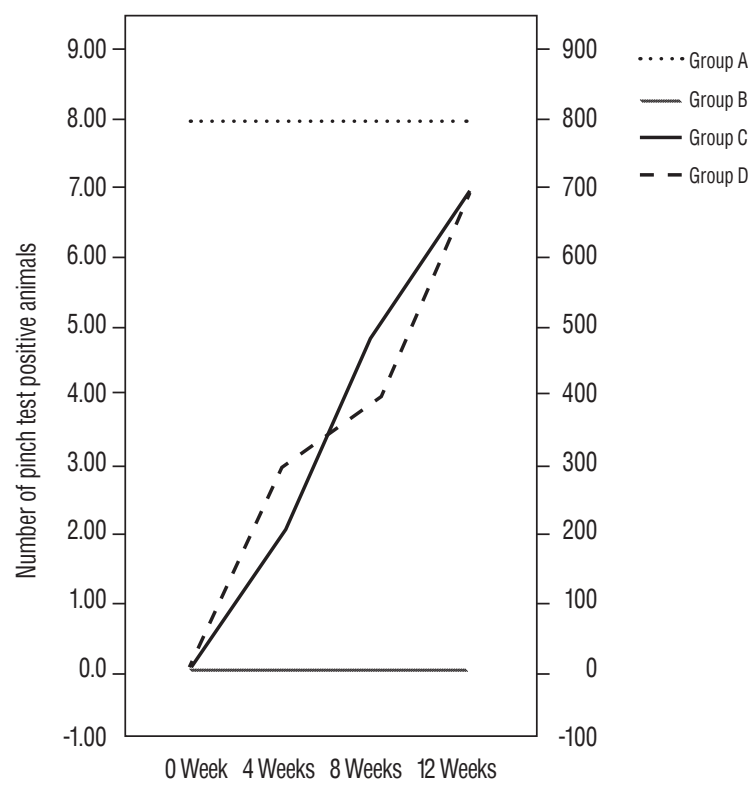

Fig. 3. The number of pinch test positive rats in different time intervals. were significantly lower than that of the sham operated group. There was also a significant difference between groups B and $\mathrm{C}(p=0.05)$. In contrast, the mean value for group D was significantly higher than for group B $(p=0.045)$, but the difference between group $\mathrm{C}$ and group D was not significant $(p=0.558)$

(Figs. 4-6).

\section{DISCUSSION}

ETE anastomosis is the golden standard in peripheral nerve injuries $^{18)}$. On the other hand, if there is a defect between the proximal and distal ends in nerve lacerations, ETS anastomosis is commonly performed for surgical repair. This technique allows the repair of the nerve despite the defect and enables functionality by the help of neighboring nerve. Technique has first been described by Ballance et al. and popularized by Viterbo ${ }^{21,22)}$. Previously, while ETS anastomosis technique was used for surgical repair of motor nerves, more recently it has been performed for the repair of sensory nerves as well ${ }^{13,19)}$.

Although it is said that sensory and motor recoveries are limited, their clinical practice has recently become popu$\operatorname{lar}^{9,11,14,16)}$. It is observed that ETS anastomosis for the repair of sensory nerves is rather performed in the end organs like hands and feet, which have a high level of tactile perception ${ }^{13,19)}$.

Previous research has demonstrated that peripheral nerve axons show improved recovery following trauma, compared

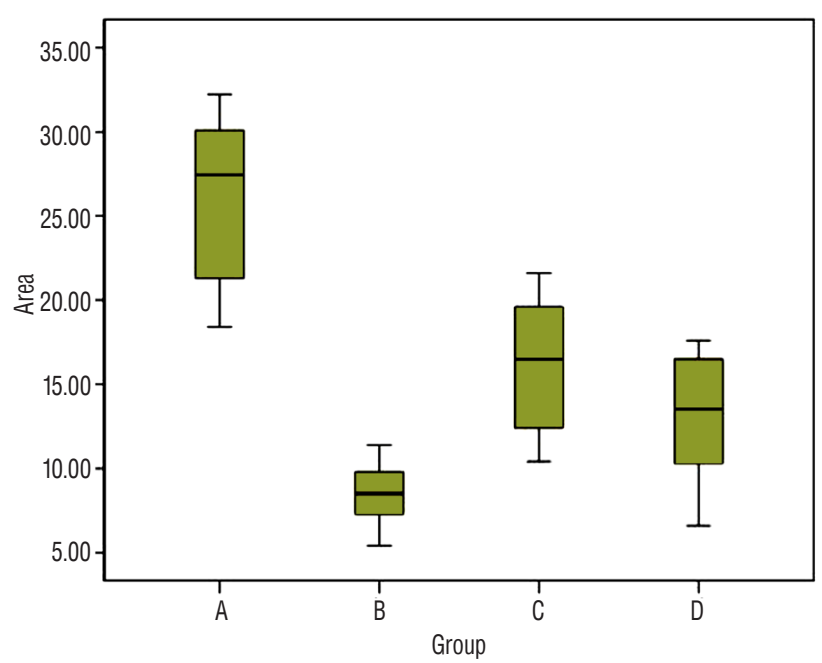

Fig. 4. A box plot summarizing the mean values of positively stained nerve endings in each group. 
to central nerve axons. After destruction of the myelin sheath by macrophages, schwann cell columns are formed at the distal end of the nerve lesion and the regenerated axons then follow the schwann cell surfaces. Previous research indicates that neurotrophic hormones released from schwann cells at the distal nerve stump induce peripheral nerve regeneration ${ }^{8,10)}$. Although there have been many studies of peripheral nerve regeneration, research has not previously been conducted on NCAM expression in free nerve endings under conditions of ETS coaptation repair in a pure sensory nerve innervation area.

NCAM (CD56) is a calcium-independent adhesion molecule, and it is also the first cellular adhesion molecule, with expression by all neurons at very early developmental stages. Additionally, NCAM is expressed by glial, skeletal muscle, and natural killer (NK) cells. The molecules bind homophilically to neighboring cells and have various functions during neural development, including cohesion of developing neurites and
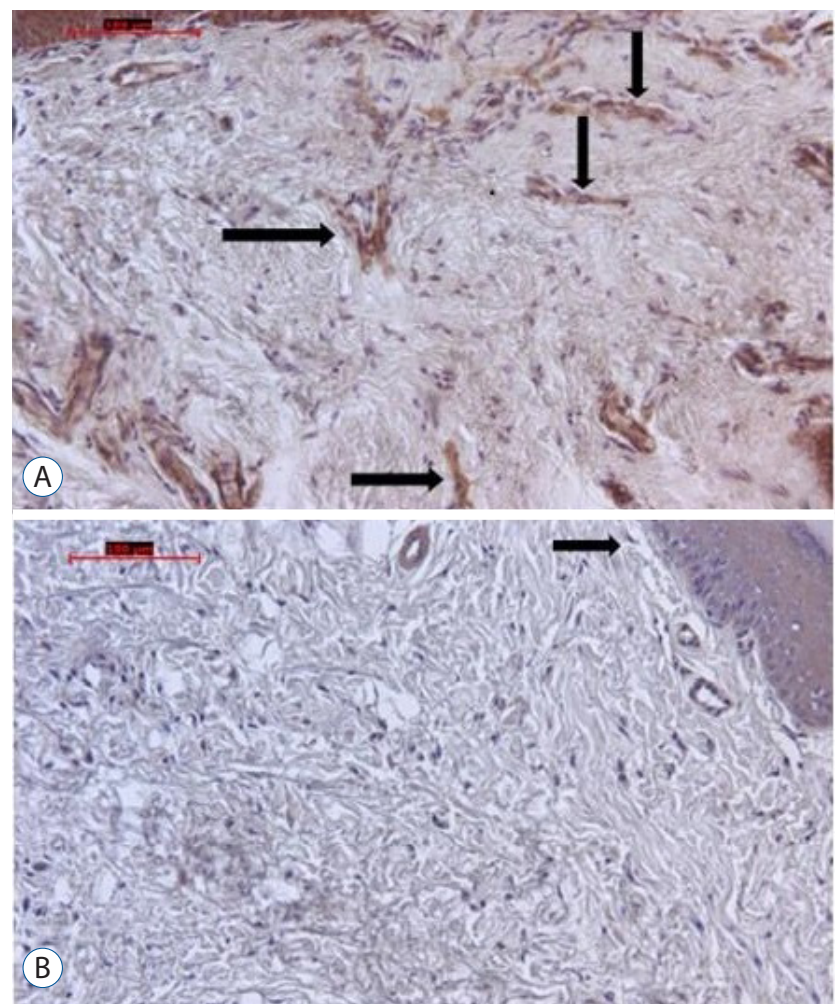

Fig. 5. A : On the micrograph of group A the arrows point anti-NCAM positive free nerve endings (original magnification, $\times 20$, indirect immunohistochemistry). B : On the micrograph of group B the arrow points anti-NCAM positive free nerve ending (original magnification, $\times 20$, indirect immunohistochemistry). More stained free nerve endings are seen on group A than in group B. NCAM : neural cell adhesion molecule. providing connections during neuronal outgrowth. The adhesive properties of NCAM in development and plasticity are regulated by carbohydrates and polysialic acid (PSA). Evidence suggests that posttranslational insertion of PSA (NCAMPSA), leads to synaptic plasticity and nerve regeneration mechanisms during learning and memory ${ }^{15}$. Additionally, NCAM is used to detect some types of tumors by immunohistochemistry. NCAM exhibits pleotropic expression, including in NK cells, activated T cells, as well as cerebral, cerebellar, and neuroendocrine tissues ${ }^{5}$.

The aim of this study was to test the efficacy of ETS anastomosis of a purely sensory nerve to a neigbouring nerve with motor functions by examining the expression of NCAM. In the present study, a significant difference in NCAM expressi-

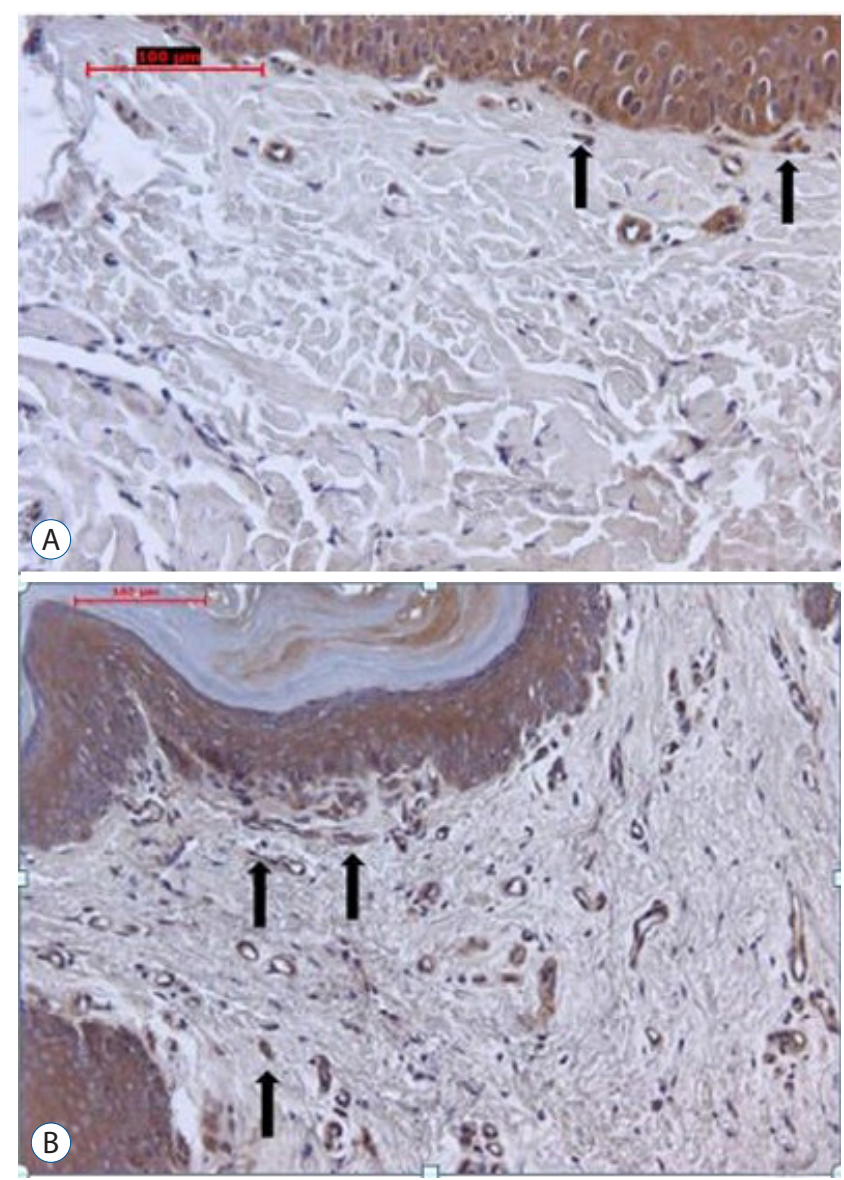

Fig. 6. A : On the micrograph of group $C$ the arrows point anti-NCAM positive free nerve endings (original magnification, $\times 20$, indirect immunohistochemistry). $B$ : On the micrograph of group $D$ the arrows point anti-NCAM positive nerve endings (original magnification, $\times 20$, indirect immunohistochemistry). More stained free nerve ending are seen on group C than in group D. NCAM : neural cell adhesion molecule. 
on was observed between tissues from the control group A rats with innervated dermatomal areas and the experimental group B rats with denervated dermatomal areas. NCAM expression in group A was significantly higher than in group B. Axonal continuity occurred in group A, whereas the axonal continuity was experimentally disrupted in group B. Thus, neural continuity and NCAM expression was reduced in the denervated dermatomal areas. Previous studies have also demonstrated functions of NCAM, including axonal growth, cellular migration, synaptic plasticity, neuronal growth, cohesion of developing neurites, and providing connections during neuronal outgrowth and synaptogenesis ${ }^{3,12)}$.

The results of the present study concur with these previous findings. Our sensory function assessment, the pinch test, resulted in positive responses from all rats in group A, and negative responses from all rats in group B. As predicted, the results did not change over time, indicating successful denervation in group B. These results support that the sural nerve innervation area described in previous studies is accurate, and verify that the sensory function assessment yields negative responses ${ }^{2,6,7)}$. Statistical analyses indicated a significant difference in NCAM expression between group A and both group C and group D. However, there was no significant difference between group $\mathrm{C}$ and group $\mathrm{D}$. The results show that reinnervation pattern is similar in the groups $\mathrm{C}$ and $\mathrm{D}$ in terms of NCAM expression.

There was no significant difference in the sensory function assessment at 12 weeks between group A and groups C or D, however, earlier recovery occurred in the ETE coaptation reinnervation group (group C). Reinnervation was achieved in both groups (C and D) according to the sensory function assessment, and NCAM expression was not significantly different between groups $\mathrm{C}$ and $\mathrm{D}$.

These results support the hypothesis that significant differences in NCAM expression at free nerve endings occur in the corresponding dermatomal area, between the group with innervated skin areas and the deinnervated groups.

ETE repair is the gold standard in peripheral nerve repair and it has been suggested as an alternative method when it is not possible to perform the ETE technique ${ }^{18)}$. In our study, the ETS coaptation method resulted in significant changes in NCAM expression. In addition, we observed improvements in the sensory function assessment of both reinnervated groups (C and D).
While some studies reported that motor axons have enhanced regeneration capacity compared to sensory axons when ETS nerve repair is performed, others reported that sensory axons have greater regenerative capacity ${ }^{1,17}$. Our success with ETS repair in the present study further supports this capacity of sensory axons.

The main limitation of our study is that we did not demonstrate the source of regenerating sensory axons. According to previous research, collaterally sprouting axons from an uninjured donor nerve (the tibial nerve in the present experiment) may be the regeneration source. These experiments have coapted only the distal stump of the transected nerve to the intact nerve, using an ETS technique. Alternately, the source of regeneration may be the proximal stump of the transected nerve, which was coapted to the intact nerve together with the distal stump. Examples of such experiments are present in the literature. In the present experiment, both the proximal and distal stumps of the transected sural nerve were coapted to the tibial nerve. Therefore, the observed functional and histological recovery may have resulted from the sensory axons sprouting from the proximal end to the distal end, or from the axons sprouting from the tibial nerve. Regardless of the source, the results of our study confirm ETS anastomosis of a transected nerve to an intact nerve as an efficacious nerve repair technique.

\section{CONCLUSION}

The present results, together with previous studies, demonstrate that neurotrophic agents are associated with the efficacy of peripheral nerve repair techniques and that further research is therefore required. Furthermore, the results of our study confirm that ETS anastomosis of a transected nerve to an intact nerve is equally efficacious to ETE anastomosis for sensory nerve repairs.

\section{References}

1. al-Qattan $M M$, al-Thunyan $A$ : Variables affecting axonal regeneration following end-to-side neurorrhaphy. Br J Plast Surg 51 : 238-242, 1998

2. Bajrović F, Sketelj $J$ : Extent of nociceptive dermatomes in adult rats is not primarily maintained by axonal competition. Exp Neurol 150 : 115 121, 1998 
3. Cunningham BA, Hemperly JJ, Murray BA, Prediger EA, Brackenbury R, Edelman GM : Neural cell adhesion molecule: structure, immunoglobulin-like domains, cell surface modulation, and alternative RNA splicing. Science 236 : 799-806, 1987

4. Dvali LT, Myckatyn TM : End-to-side nerve repair: review of the literature and clinical indications. Hand Clin 24 : 455-460, vii, 2008

5. Jensen $M$, Berthold $F$ : Targeting the neural cell adhesion molecule in cancer. Cancer Lett $258:$ 9-21, 2007

6. Kovacic U, Bajrović F, Sketelj J : Recovery of cutaneous pain sensitivity after end-to-side nerve repair in the rat. J Neurosurg 91 : 857-862, 1999

7. Kovacic U, Tomsic M, Sketelj J, Bajrović FF : Collateral sprouting of sensory axons after end-to-side nerve coaptation--a longitudinal study in the rat. Exp Neurol $203:$ 358-369, 2007

8. Mccallister WV, Tang P, Smith J, Trumble TE : Axonal regeneration stimulated by the combination of nerve growth factor and ciliary neurotrophic factor in an end-to-side model. J Hand Surg Am 26 : 478-488, 2001

9. Noah EM, Williams A, Jorgenson C, Skoulis TG, Terzis JK : End-to-side neurorrhaphy: a histological and morphometric study of axonal sprouting into an end-to-side nevre graft. J Reconstr Microsurg 13 : 99106, 1997

10. Okajima S, Terzis JK : Ultrastructure of early axonal regeneration in an end-to-side neurorrhaphy model. J Reconstr Microsurg 16 : 313-326; discussion 323-326, 2000

11. Pannucci C, Myckatyn TM, Mackinnon SE, Hayashi A : End-to-side nerve repair: review of the literature. Restor Neurol Neurosci $25:$ 45-63, 2007

12. Pillai-Nair N, Panicker AK, Rodriguiz RM, Gilmore KL, Demyanenko GP, Huang JZ, et al. : Neural cell adhesion molecule-secreting transgenic mice display abnormalities in GABAergic interneurons and alterations in behavior. J Neurosci 25 : 4659-4671, 2005

13. Rinker BD, Ingari JV, Greenberg JA, Thayer WP, Safa B, Buncke GM : Outcomes of short-gap sensory nerve injuries reconstructed with pro- cessed nerve allografts from amulticenter registry study. J Reconstr Microsurg 31 : 384-390, 2015

14. Shah MH, Kasabian AK, Karp NS, Kolker AR, Dublin BA, Zhang L, et al. Axonal regeneration through an autugeneous nerve bypass: an experimental study in rats. Ann Plast Surg 38 : 408-414; discussion 414-415, 1997

15. Stoenica L, Senkov O, Gerardy-Schahn R, Weinhold B, Schachner M, Dityatev $A$ : In vivo synaptic plasticity in the dentate gyrus of mice deficient in the neural cell adhesion molecule NCAM or its polysialic acid. Eur J Neurosci 23 : 2255-2264, 2006

16. Tarasidis G, Watanabe O, Mackinon SE, Strasberg SR, Haughey BH, Hunter DA : End-to-side neororrhaphy resulting in limited sensory axonal regeneration model. Ann Otol Rhinol Laryngol 106 : 506-512, 1997

17. Tham SKJ, Morrison WA : Motor collateral sprouting through an end-toside nerve repair. J Hand Surg Am 23 : 844-851, 1998

18. Thomas MB : Nerve Repair and Grafting in Green DP, Hotchkiss RN, Pederson WC (eds) : Green's Operative Hand Surgery, ed 6. Philadelphia : Churchill Livingstone, 1999, pp1381-1404

19. Tos P, Colzani G, Ciclamini D, Titolo P, Pugliese P, Artiaco S : Clinical applications of end-to-side neurorrhaphy: an update. Biomed Res Int $2014: 646128,2014$

20. Viterbo F, Amr AH, Stipp EJ, Reis FJ : End-to-side neurorrhaphy: past, present, and future. Plastic Reconstr Surg 124(6 Suppl) : e351e358, 2009

21. Viterbo F, Trindade JC, Hoshino K, Mazzoni Neto A : End-to-side neurorrhaphy with removal of the epineurialsheat: an experimental study in rats. Plast Reconstr Surg 94 : 1038-1047, 1994

22. Viterbo F, Trindade JC, Hoshino KH, Mazzoni A: Two end-to-side neurorrhaphies and nerve graft with removal of the epineural sheath: experimental study in rats. Br J Plast Surg 47 : 75-80, 1994 